\title{
SIMULATION AND ANALYSIS OF PHOTOGRAMMETRIC UAV IMAGE BLOCKS: INFLUENCE OF CAMERA CALIBRATION ERROR
}

\author{
Y. Zhou ${ }^{1 *}$, E. Rupnik ${ }^{1}$, C. Meynard ${ }^{1}$, C. Thom ${ }^{1}$, M. Pierrot-Deseilligny ${ }^{1}$ \\ ${ }^{1}$ LaSTIG, IGN, ENSG, University Paris-Est, F-94160 Saint-Mande, France \\ (Yilin.Zhou, Marc.Pierrot-Deseilligny)@ensg.eu, (Ewelina.Rupnik, Christophe.Meynard, Christian.Thom)@ign.fr
}

\author{
Commission I, ICWG I/II
}

KEY WORDS: UAV, camera calibration, aerial photogrammetry, corridor mapping, bundle block adjustment (BBA), thermal effect

\begin{abstract}
:
Unmanned aerial vehicles (UAV) are increasingly used for topographic mapping. The camera calibration for UAV image blocks can be performed a priori or during the bundle block adjustment (self-calibration). For an area of interest with flat, corridor configuration, the focal length of camera is highly correlated with the height of camera. Furthermore, systematic errors of camera calibration accumulate on the longer dimension and cause deformation. Therefore, special precautions must be taken when estimating camera calibration parameters. In this paper, a simulated, error-free aerial image block is generated. error is then added on camera calibration and given as initial solution to bundle block adjustment. Depending on the nature of the error and the investigation purpose, camera calibration parameters are either fixed or re-estimated during the bundle block adjustment. The objective is to investigate how certain errors in the camera calibration impact the accuracy of 3D measurement without the influence of other errors. All experiments are carried out with Fraser camera calibration model being employed. When adopting a proper flight configuration, an error on focal length for the initial camera calibration can be corrected almost entirely during bundle block adjustment. For the case where an erroneous focal length is given for pre-calibration and not re-estimated, the presence of oblique images limits the drift on camera height hence gives better camera pose estimation. Other than that, the error on focal length when neglecting its variation during the acquisition (e.g., due to camera temperature increase) is also investigated; a bowl effect is observed when one focal length is given in camera pre-calibration to the whole image block. At last, a local error is added in image space to simulate camera flaws; this type of error is more difficult to be corrected with the Fraser camera model and the accuracy of 3D measurement degrades substantially.
\end{abstract}

\section{INTRODUCTION}

The derivation of geospatial information from unmanned aerial vehicles (UAV) is becoming increasingly ubiquitous (Nex , Remondino, 2014). By applying proper processing, a 3D scene can be reconstructed from aerial images with high accuracy. The bundle block adjustment is a basic tool for photogrammetric scene reconstruction. In essence, the procedure consists of identifying common feature points between overlapping images and recovering their poses (i.e. positions and orientations) at first in a relative coordinate system, followed by the georeferencing phase with the help of, e.g. ground control points (GCP), or the camera positions measured with global navigation satellite system (GNSS) (Heipke et al., 2002, Cramer et al., 2000). Camera calibration parameters can be considered pre-calibrated and constant, or their values are re-estimated in the self-calibrating bundle block adjustement. (Westoby et al., 2012, Fonstad et al., 2013).

In corridor mapping, the deformation caused by the accumulation of camera calibration errors often appears; this phenomenon is called doming effect or bowl effect (James, Robson, 2014). Hence, precautions should be taken to determining properly interior orientation parameters. If the area of interest is largely flat, the estimation of the focal length may be inaccurate due to its high correlation with camera height. Cross flight patterns, different flight heights and the addition of oblique images may render the estimation more reliable (Zhou et al., 2018) nonethe-

\footnotetext{
*Corresponding author
}

less in a corridor configuration, flight configuration is limited and cross flight patterns are not easy to achieve.

In this paper, we generate a simulated, error-free aerial image block which is of flat, corridor configuration. Different types of errors are added on the camera calibration; then the erroneous camera calibration is given as an initial solution to the bundle block adjustment. Depending on the nature of the error and the investigation purpose, camera calibration parameters are either fixed or re-estimated during the bundle block adjustment. The impacts of each type of error on camera calibration and photogrammetric accuracy are investigated.

\section{DATA GENERATION AND RESEARCH DESIGN}

\subsection{Generation of a simulated dataset}

A real aerial image block is employed for the generation of a simulated, error-free image block. The acquisition field consists of a north-south oriented dike of $200 \mathrm{~m}$, which presents a flat, corridor configuration. A total of four flights are performed: one flight of 3 strips at $50 \mathrm{~m}$ with nadir pointing camera, one flight of 1 strip at $30 \mathrm{~m}$ with nadir pointing camera, one flight of 1 strip at $70 \mathrm{~m}$ with nadir pointing camera and one flight of 3 strips at $50 \mathrm{~m}$ with oblique pointing camera. See Figure 1 and Table 1 for more details on conducted flights.

The original camera poses and camera calibration (camera distortion model: Fraser (Fraser, 1997)) are considered as ground truth and the simulated dataset is generated basing on it. A 


\begin{tabular}{|c|c|c|c|c|}
\hline Flight & 1 & 2 & 3 & 4 \\
\hline $\mathrm{Nb}$ of images & 42 & 12 & 15 & 44 \\
\hline Height (m) & 50 & 30 & 70 & 50 \\
\hline Orientation & nadir & nadir & nadir & oblique \\
\hline $\mathrm{Nb}$ of strips & 3 & 1 & 1 & 3 \\
\hline $\begin{array}{l}\text { Overlap } \\
(\%)\end{array}$ & \multicolumn{4}{|c|}{80} \\
\hline camera focal length & \multirow{2}{*}{\multicolumn{4}{|c|}{$\frac{35 \mathrm{~mm} / 5510 \text { pixels }}{6.4}$}} \\
\hline camera sensor pixel size $(\mu \mathrm{m})$ & & & & \\
\hline camera sensor size (pixel) & \multicolumn{4}{|c|}{$5120 \times 3840$} \\
\hline GSD (mm) & 10 & 6 & 14 & 10 \\
\hline
\end{tabular}

Table 1. Information of flights

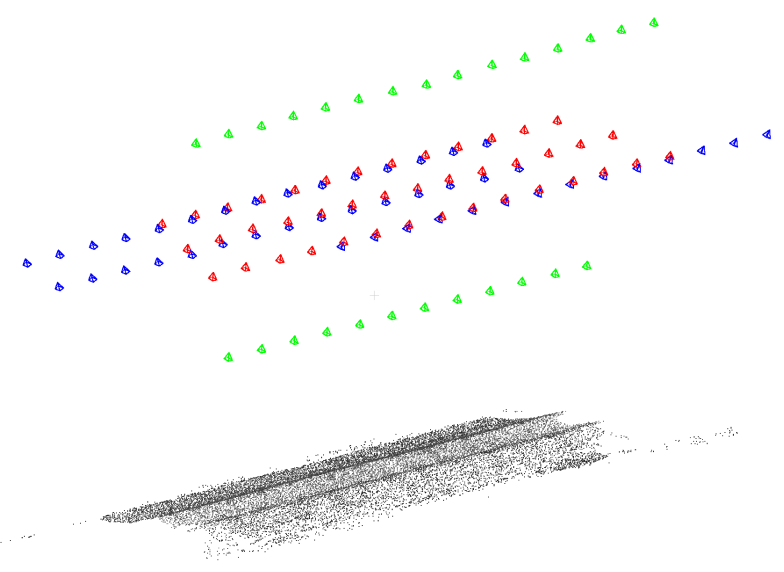

Figure 1. Illustration of flights: nadir flight of 3 strips at $50 \mathrm{~m}$ (in red), oblique flight of 3 strips at $50 \mathrm{~m}$ (in blue) and nadir flights of 1 strip at 30/70m (in green).

set of 3D points is obtained by performing pseudo-intersection with original tie points. Afterwards, this set of 3D points is reprojected in all images for the generation of simulated tie points. Note that the tie points generated this way intersect perfectly. A subset of these 3D points also serve as GCPs/CPs, their corresponding reprojections on images will serve as image measurements of GCPs/CPs. Figure 2 depicts how simulated dataset is generated.

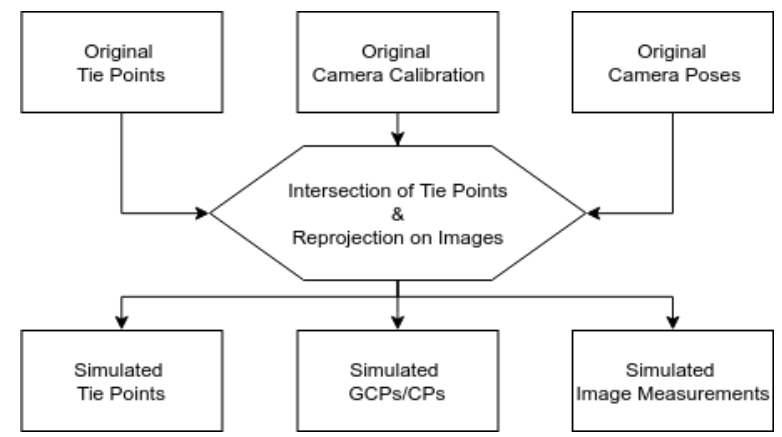

Figure 2. Workflow of the generation of simulated dataset.

The simulated dataset consists of simulated tie points, simulated GCPs/CPs and their image measurements, original camera calibration and original camera poses. For the simulated dataset, the RMS of its reprojection error on images equals to $38.4 \mathrm{~nm} / 0.006$ pixels, the RMS of its residuals on CPs is $3.1 \mathrm{~nm}$, these two indicate a good consistency among tie points, camera poses, camera calibration and GCPs/CPs. It also indicates the highest accuracy one can obtain with this simulated dataset.

\subsection{Error addition and result evaluation}

According to the research purposes, different errors are generated and added on respective observations. The photogrammetric processing is performed with a free, open-source photogrammetric software MicMac (Rupnik et al., 2017).

A bundle block adjustment is carried out with tie points as observations; camera calibration and camera poses are given as initial solutions. Depending on the nature of the error and the investigation purpose, camera calibration parameters are either fixed or re-estimated during the bundle block adjustment. Specifications will be given for each case. Once bundle block adjustment is done, ten well-distributed GCPs are employed for the determination of a 3D similarity and the reconstructed scene is transformed into an absolute coordinate system. The accuracy of the 3D scene will be evaluated on the RMS of residuals on five thousand well-distributed CPs. Figure 3 shows the workflow of error addition and result evaluation.

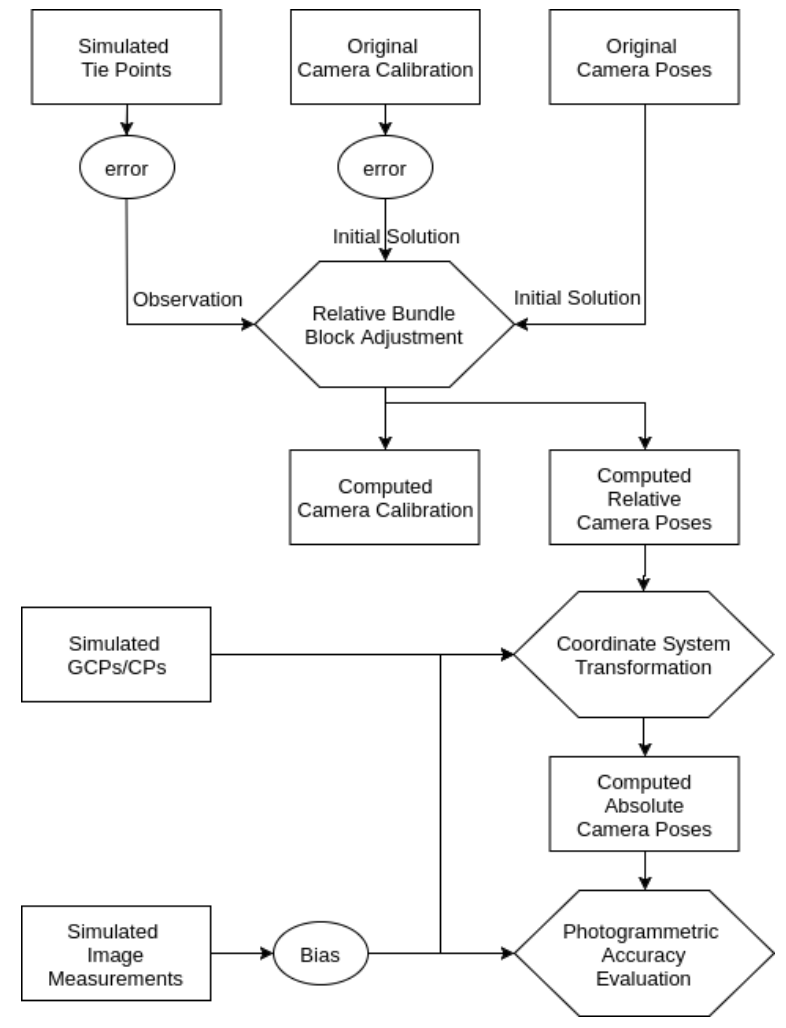

Figure 3. Workflow of error addition and result evaluation.

\section{EXPERIMENTS AND RESULTS}

In this section, we present two types of error on the focal length and one type of error simulating camera flaws. Each type of error is simulated and the respective impacts on camera calibration estimation and 3D measurement accuracy are investigated.

\subsection{Error coming from false camera calibration}

\section{Erroneous focal length}

To investigate how camera poses and 3D accuracy vary with error on focal length for camera pre-calibration, an error which varies from -50 pixels to +50 pixels is added on the focal length (original value: 5510 pixels). During the bundle block adjustment, the focal length is fixed while other camera calibration 
parameters are freed and re-estimated. Two cases have been investigated: in the first case, images of all flights (flight 1-4) are introduced to the bundle block adjustment; in the second case, only nadir images of three different heights (flight 1-3) are introduced. Figure 4 depicts the variation of residuals on CPs and of camera average height for both cases.

One can see that in both cases, the residuals increase linearly with the error on focal length. The sign of the error does not affect the amplitude of residual RMS. For the case with only nadir images, the RMS is much smaller than in the other case. Secondly, the change of average camera height also changes linearly with the applied error; with only nadir images, the change of average camera height is greater. It can be explained by the fact that, during the bundle block adjustment the RMS is minimized by drifting the height of the camera, accuracy on camera heights degrades for the trade-off of higher 3D accuracy. The presence of oblique images adds constraints on camera heights therefore the obtained camera poses are closer to theoretical values whereas the $3 \mathrm{D}$ accuracy is compromised.

In the case where the focal length of the camera calibration is erroneous, one can expect a homothetic form of error in image space (tie points and image measurements). To verify this assumption as well as to see if this error can be corrected during bundle block adjustment, a homothetic error is added in image space. The homothetic form of error can be expressed by the equation

$$
b=\lambda \cdot \overrightarrow{C Q}
$$

where: $\quad b$ is the error added to images;

Point $C$ is the center point of the homothetic error; Point $Q$ is the point in image to add error to; $\lambda$ is the scaling factor, here equals to 0.005 .

Since for Fraser camera distortion model, the principal point (PP) and the distortion center (DC) are considered different, the same homothetic error is added on images and centered on PP and DC, respectively. The original values of PP and DC are $[2539.75,1945.80]$ and $[2537.97,1955.92]$; coordinates are expressed in pixel. One can see that the PP and the DC are close yet different. Figure 5 gives an example of the described homothetic error centered on PP. The error is of 0 pixel at its center and increases with the radial distance w.r.t its center; the maximum of the error centered on PP is 16.15 pixels.

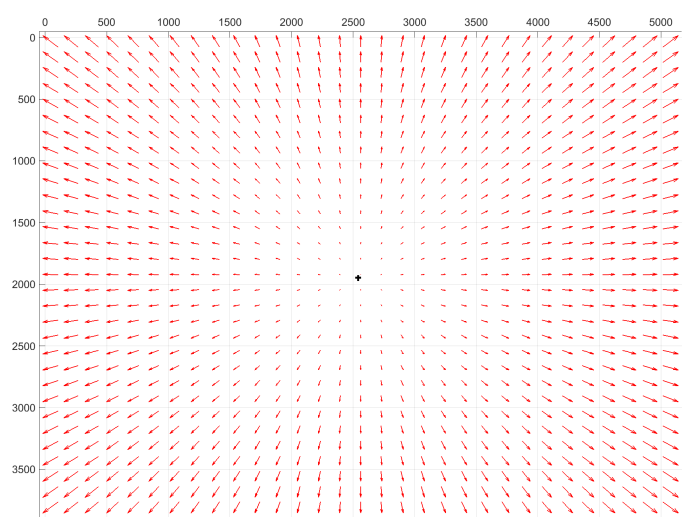

Figure 5. The homothetic error centered on PP. The direction and the magnitude of vectors represent the direction and the magnitude of added error at the origin of vectors. The cross in black indicates the position of PP.
During the bundle block adjustment, erroneous tie points serve as observations and all camera calibration parameters are freed and re-estimated. Table 2 shows the variation of camera calibration parameters in each case.

\begin{tabular}{ccccc}
\hline & & Original & Bias on PP & Bias on DC \\
\hline \multirow{2}{*}{ PP } & $\mathrm{x}$ & 2539.75 & $2539.75 \pm 0.005$ & $2539.76 \pm 0.005$ \\
& $\mathrm{y}$ & 1945.80 & $1945.80 \pm 0.005$ & $1945.75 \pm 0.005$ \\
\hline \multirow{2}{*}{ DC } & $\mathrm{x}$ & 2537.97 & $2537.96 \pm 0.004$ & $2537.97 \pm 0.004$ \\
& $\mathrm{y}$ & 1955.92 & $1955.96 \pm 0.004$ & $1955.92 \pm 0.004$ \\
\hline $\mathrm{F}$ & & 5510.09 & $5537.64 \pm 0.001$ & $5537.64 \pm 0.001$ \\
\hline
\end{tabular}

Table 2. Variation of primary camera calibration parameters before and after the addition of homothetic error. The parameters are expressed in pixel.

One can observe that in both cases, when the error is centered on one point (either PP or DC), this point remains unchanged after the bundle block adjustment while the other point varies slightly (the variation is negligible). On the contrary, in both cases, the focal length varies significantly from $5510.09 \mathrm{px}$ to $5537.64 \mathrm{px}$. It is due to the fact that a homothetic form of error is, in a manner of speaking, an error on focal length. The focal length is corrected during the bundle block adjustment. The ratio of the variation of focal length equals to 0.0049 and is quite close to the value of $\lambda=0.005$, the scaling factor of the error. Table 3 gives statistics on the variation of camera distortion correction for both cases, the difference between two cases is insignificant.

\begin{tabular}{lcccc}
\hline & min & max & mean & std \\
\hline Bias on PP & 0.02 & 14.85 & 8.49 & 3.21 \\
Bias on DC & 0.02 & 14.86 & 8.49 & 3.21 \\
\hline
\end{tabular}

Table 3. Statistics of the variation of camera distortion correction. The parameters are expressed in pixel.

To keep the article concise, in the following part, figures are only given for the case where the error is centered on PP. Figure 6 depicts the spatial distribution of variations on camera calibration, the variation of camera distortion correction is quite close to the added error. For the reconstructed 3D scene, the residuals on CPs are in the order of $\mu \mathrm{m}$, the RMS of image reprojection error equals to 0.01 pixels. It means the added homothetic error is compensated almost entirely by the camera calibration.

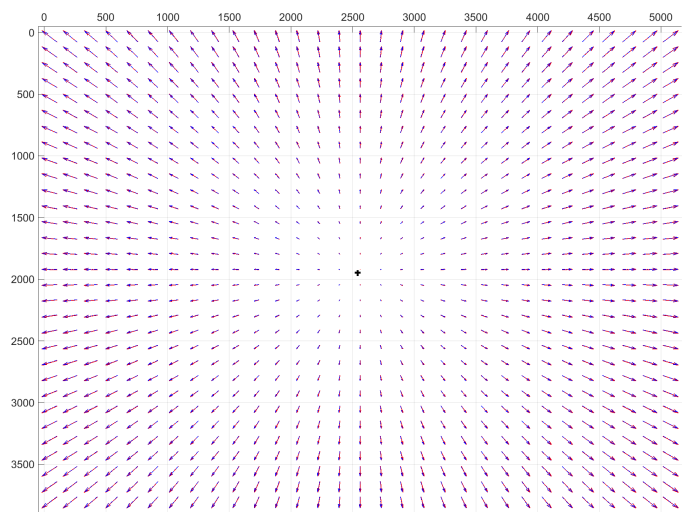

Figure 6. In red: the homothetic error centered on PP as shown in Figure 5. In dashed blue: the variation of camera distortion correction. The same plotting conventions as in Figure 5 are applied 

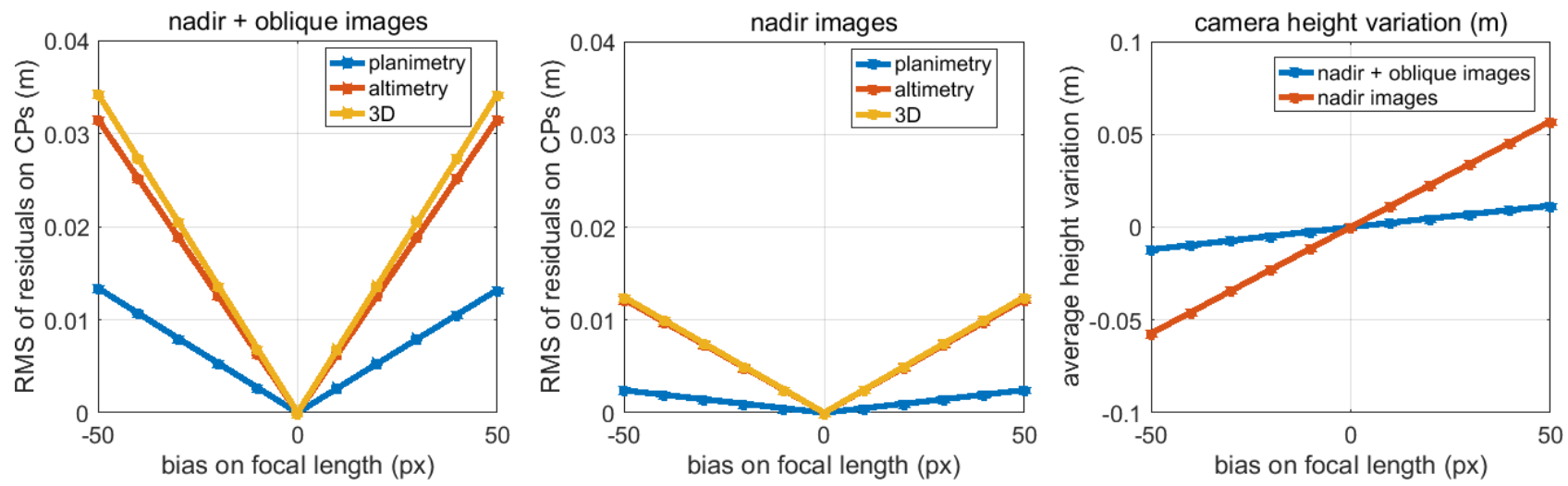

Figure 4. Variation of residuals on CPs and camera height with error on the focal length. Left: variation of residuals on CPs with error on focal length when all images (flight 1-4) are introduced to bundle block adjustment. Middle: variation of residuals on CPs with error on focal length when only nadir images (flight 1-3) are introduced to bundle block adjustment. Right: variation of average camera height with error on focal length.

\section{Gradually varied focal length}

In this part, another possibility of false focal length is simulated: the camera focal length varies gradually during the acquisition whereas this variation is not taken into account during the processing. The variation of focal length is often encountered when the acquisition is carried out with a high frame rate. The internal temperature of the camera can increase significantly and leads to a gradual change of the effective focal length (Daakir et al., 2019). However, during photogrammetric processings, one often assume that the camera calibration parameters do not vary and one camera calibration is applied to the whole dataset.

To simulate this problem, one camera calibration is calculated (all parameters are freed and re-estimated) per image with simulated tie points and original camera poses. The focal length is manually changed so that it varies in an exponential way during the acquisition. The variation of focal length can be expressed by the equation

$$
f^{\prime}(n)=f *(1+b)^{n}
$$

where: $\quad n$ is the image index

$f$ is the original focal length;

$1+b$ is the base, here $b$ equals to $1 \mathrm{e}-5$;

$f^{\prime}(n)$ is the modified focal length for image $n$;

Here we assume that four flights are carried out one after another without break, following the order: oblique flight of 3 strips at $50 \mathrm{~m}$, nadir flight of 3 strips at $50 \mathrm{~m}$, nadir flight of 1 strip at $30 \mathrm{~m}$ then nadir flight of 1 strip at $70 \mathrm{~m}$. The focal length of the last image (5516.43 pixels) is 6.17 pixels greater then that of the first image (5510.26 pixels). With $b$ being much smaller than 1 in the equation (2), the variation of the focal length is approximately linear. According to the relation between the focal length and the camera internal temperature given by (Daakir et al., 2019), an increase of 6.17 pixels corresponds to a temperature increase of around $80^{\circ} \mathrm{C}$ for a focal length of $35 \mathrm{~mm}$ as in our experiments. In real cases, the temperature increase of the camera is more likely to be around $20^{\circ} \mathrm{C}-40^{\circ} \mathrm{C}$. The increase of the temperature is exaggerated in the simulation to render the impact more visible. Figure 7 depicts the variation of the focal length during the acquisition.

Tie points and GCPs/CPs image measurements are regenerated based on original camera poses and modified camera calibra-

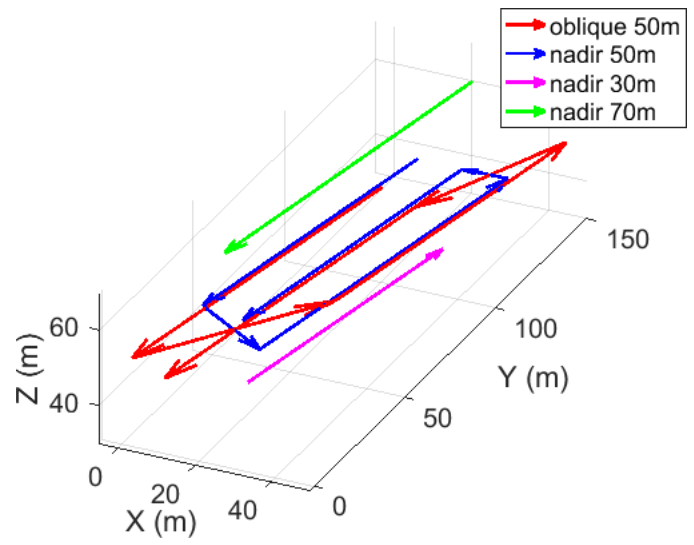

Figure 7. Flights are considered conducted in the order of legend list. The focal length increases in the direction of vectors.

tions. After that, the dataset is processed with one camera calibration for all images as which is usually done in the practice. During the bundle block adjustment, no elimination is performed on tie points, all camera calibration parameters are freed and re-estimated. The estimated focal length when all images are considered to share the same camera calibration equals to 5512.27 pixels.

Residuals on CPs are given in Figure 8 and Table 4. Figure 8 depicts the spatial distribution and the histogram of residuals on CPs. One can observe that there exists a slight bowl effect, the two ends of the dike have important residuals along $+z$ axis and the middle part of the dike has residual along $-z$ axis. The pattern of the residuals on CPs shown in Figure 8 is a consequence of the focal length variation pattern. The mixture of nadir images of different altitudes and oblique images can be the reason for the asymmetric residual pattern. The magnitude of residuals on altimetry is less than $1 \mathrm{~mm}$ since the acquisition field is small (200m). However, when processing large scale datasets, the degradation of accuracy can be important.

\begin{tabular}{ccc}
\hline \multicolumn{3}{c}{ Gradually varied focal length } \\
\hline planimetry $(\mathrm{mm})$ & altimetry $(\mathrm{mm})$ & 3D $(\mathrm{mm})$ \\
$0.26 \pm 0.19$ & $0.92 \pm 0.77$ & $1.0 \pm 0.74$ \\
\hline
\end{tabular}

Table 4. Statistics of the residuals on CPs. 

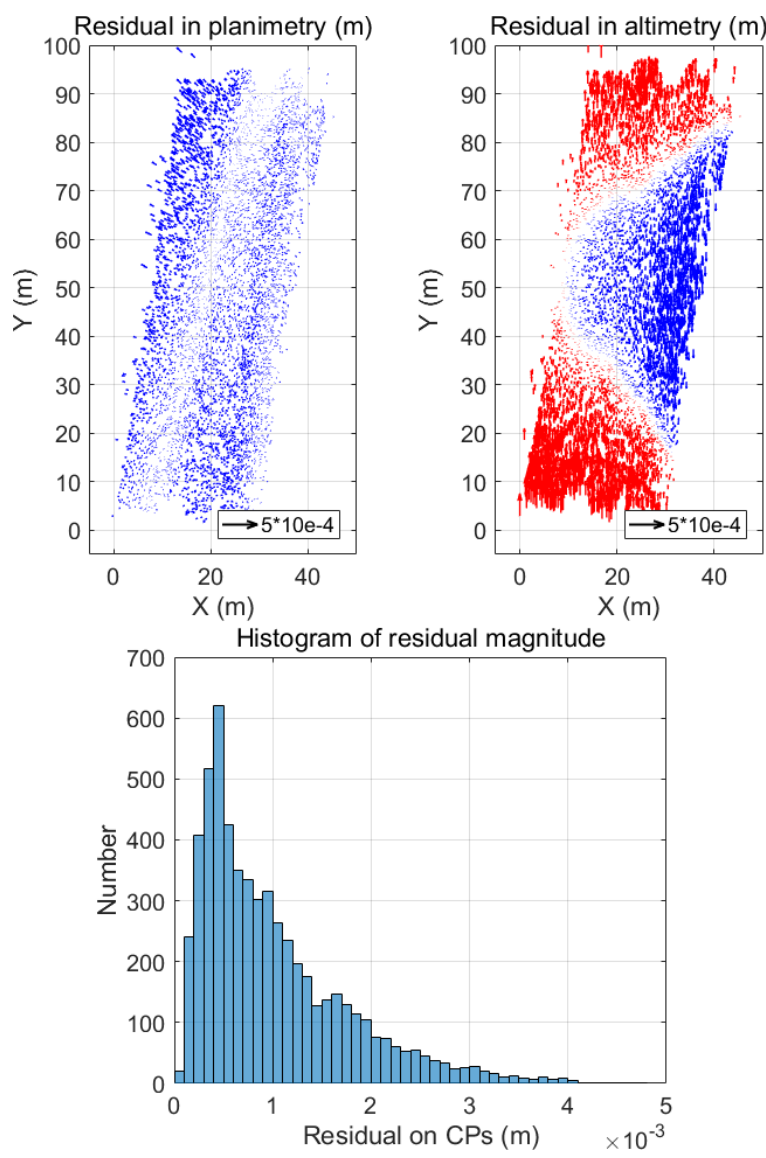

Figure 8. The spatial distribution of residuals on CPs when the variation of focal length is not taken into account. Upper left: residual on planimetry, vector direction and magnitude represent residual direction and magnitude, respectively. Upper right: residual on altimetry, vector direction and color represent the sign of residuals, upward red means positive, downward blue means negative; vector magnitude represents residual magnitude. The first two images share the same scale.

Bottom: histogram of residual magnitude.

\subsection{Bias coming from camera flaws}

In this part, an error is added to images on a small area to simulate a camera flaw (e.g. non-planarity of sensor due to incorrect gluing; optical defects of protecting glass or spectral filters). The magnitude of the error is Gaussian and can be assumed to be expressed by the equation (3):

This kind of error is difficult to be modeled with the Fraser camera distortion model. During the bundle block adjustment, no elimination is performed on tie points; all camera calibration parameters are freed and re-estimated. The variation of camera distortion correction is given in Figure 9, the statistics are given in Table 5. Compared to when a global error is added (e.g., a homothetic error centered on PP), the variation of camera distortion correction in this case is much smaller. Its geometrical form does not correspond to the applied error, either.

$$
b=\lambda \cdot e^{\frac{\|C Q\|^{2}}{\sigma^{2}}} \cdot \frac{\overrightarrow{C Q}}{\|C Q\|}
$$

where: $\quad b$ is the error added to tie points;
Point $C$ is the center of the error $C=[1000,1000]$; Point $Q$ is the tie point to add noise to;

$\lambda$ is the scaling factor, $\lambda=10$; $\sigma$ is the density, $\sigma=200$.

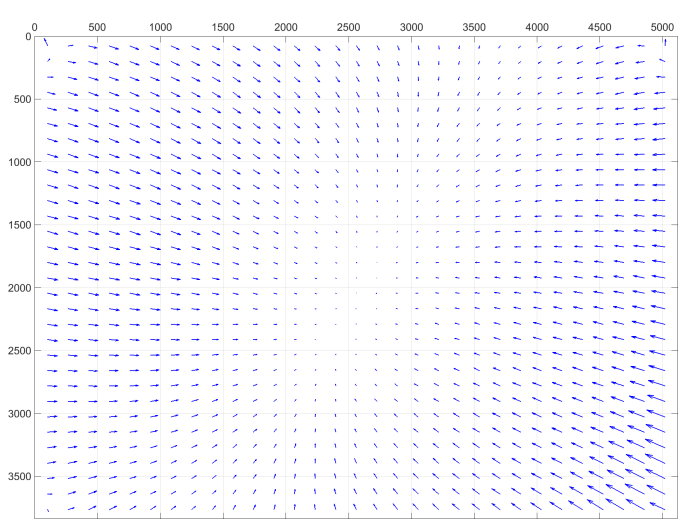

Figure 9. The variation of camera distortion correction with the local Gaussian error. The direction and the magnitude of the vectors represent the direction and the magnitude of the variation of camera distortion correction at the origin of the vector, respectively.

\begin{tabular}{cccc}
\hline \multicolumn{4}{c}{ Local Gaussian error } \\
\hline $\min$ & $\max$ & mean & std \\
0.0056 & 0.5784 & 0.1932 & 0.0996 \\
\hline
\end{tabular}

Table 5. Statistics of the variation of camera distortion correction. The results are expressed in pixel.

Residuals on CPs are given in Figure 10 and Table 6. Figure 10 gives the spatial distribution and histogram of residuals on CPs. As for the local Gaussian error, its order of residuals is the highest among all three types of errores presented above while its magnitude of error and its applied area being the smallest. The left side of the dike has anomalies which are difficult to interpret. The upper right and lower right part of the dike has more significant residuals than the middle part. The anomalies and high residuals are results of the fact that this kind of local error is difficult to be modeled by common camera calibration models such as Fraser.

\begin{tabular}{ccc}
\hline \multicolumn{3}{c}{ Local Gaussian error } \\
\hline planimetry $(\mathrm{mm})$ & altimetry $(\mathrm{mm})$ & 3D $(\mathrm{mm})$ \\
$0.89 \pm 0.82$ & $1.1 \pm 1.5$ & $1.5 \pm 1.6$ \\
\hline
\end{tabular}

Table 6. Statistics of the residuals on CPs.

\section{CONCLUSION AND DISCUSSION}

This paper studied the impact of different types of camera calibration error for a simulated aerial image block of flat, corridor configuration. The simulated, error-free aerial image block is free from the influence of errors other than those of interest.

For a camera calibration given as initial solution, the error on focal length can be corrected during the bundle block adjustment with a proper acquisition configuration. However, when an erroneous focal length is given and not re-estimated during the bundle block adjustment, camera heights drift from theoretical values to compensate for the error introduced by the erroneous focal length. The presence of oblique images limits this drift, therefore camera poses closer to theoretical values 

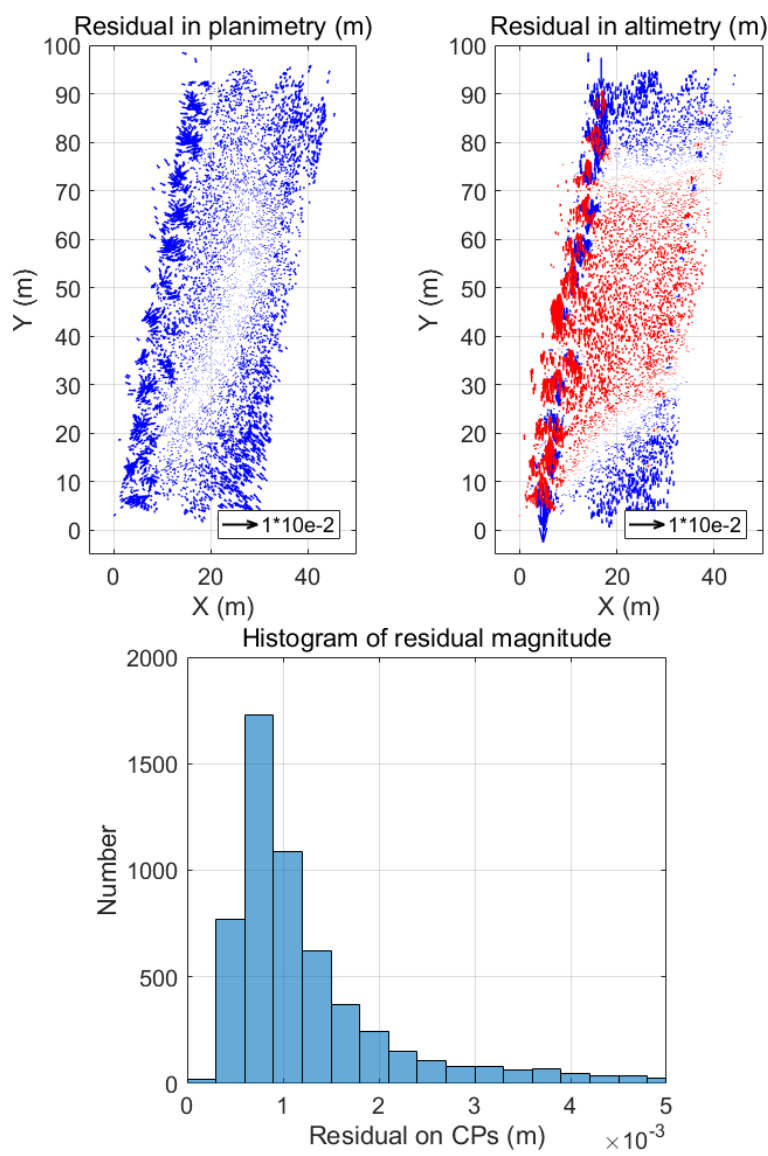

Figure 10. The spatial distribution of the residuals on CPs when a local Gaussian error is added. Upper left: residual on planimetry, vector direction and magnitude represent residual direction and magnitude, respectively. Upper

right: residual on altimetry, vector direction and color represent the sign of residuals, upward red means positive, downward blue means negative; vector magnitude represents residual magnitude. The first two images share the same scale. Bottom: histogram of residual magnitude.

are obtained whereas the accuracy of 3D measurement is compromised.

Secondly, the focal length is likely to vary during acquisitions due to the temperature change of the camera. When one camera calibration is given for all images, a slight bowl effect is observed. Though the variation of focal length is exaggerated in the experiment, it is still of importance taking it into account when a large scale image block is in concern. The relation between flight patterns and the spatial deformation of 3D models is worth further investigation.

As for a local error (e.g. introduced by camera flaws), the Fraser camera calibration model is not able to correct it properly. The 3D accuracy declines substantially though the amplitude of the error is smaller than other two errors mentioned above, and the error is applied to a limited area in image space. For future studies, empirical camera calibration models might be taken into account to see if better results could be obtained.

\section{REFERENCES}

Cramer, Michael, Stallmann, Dirk, Haala, Norbert, 2000. Direct georeferencing using GPS/inertial exterior orien- tations for photogrammetric applications. International Archives of Photogrammetry and Remote Sensing, 33, 198-205.

Daakir, Mehdi, Zhou, Yilin, Pierrot-Deseilligny, Marc, Thom, Christian, Martin, Olivier, Rupnik, Ewelina, 2019. Improvement of photogrammetric accuracy by modeling and correcting the thermal effect on camera calibration. ISPRS Jounal for Photogrammetry and Remote Sensing, 148, $142-155$

Fonstad, Mark A, Dietrich, James T, Courville, Brittany C, Jensen, Jennifer L, Carbonneau, Patrice E, 2013. Topographic structure from motion: a new development in photogrammetric measurement. Earth Surface Processes and Landforms, 38, 421-430.

Fraser, Clive S, 1997. Digital camera self-calibration. ISPRS Journal of Photogrammetry and Remote sensing, 52, 149-159.

Heipke, Christian, Jacobsen, Karsten, Wegmann, Helge, 2002. Analysis of the results of the oeepe test integrated sensor orientation. OEEPE Integrated Sensor Orientation Test Report and Workshop Proceedings, Editors, Citeseer.

James, Mike R, Robson, Stuart, 2014. Mitigating systematic error in topographic models derived from UAV and ground-based image networks. Earth Surface Processes and Landforms, 39, 1413-1420.

Nex, Francesco, Remondino, Fabio, 2014. UAV for 3D mapping applications: a review. Applied geomatics, 6, 115 .

Rupnik, Ewelina, Daakir, Mehdi, Deseilligny, Marc Pierrot, 2017. MicMac-a free, open-source solution for photogrammetry. Open Geospatial Data, Software and Standards, 2, 14 .

Westoby, MJ, Brasington, J, Glasser, NF, Hambrey, MJ, Reynolds, JM, 2012. Structure-fromMotionphotogrammetry: A low-cost, effective tool for geoscience applications. Geomorphology, 179, 300-314.

Zhou, Yilin, Rupnik, Ewelina, Faure, Paul-Henri, PierrotDeseilligny, Marc, 2018. GNSS-Assisted Integrated Sensor Orientation with Sensor Pre-Calibration for Accurate Corridor Mapping. Sensors, 18, 2783. 